\title{
Une mutation d'une protéine $G$ à l'origine de certaines ostéodystrophies d'Albright
}

Les protéines $G$ constituent une famille de protéines liant les nucléotides à guanine, qui sont des intermédiaires de la transduction des signaux à travers les membranes et qui interviennent dans des réactions multiples (m/s $n^{\circ} 8$, vol. 5, p. 562). Elles possèdent trois types de sousunités, $\alpha, \beta, \gamma$, qui forment des hétérotrimères. Ceux-ci sont nombreux du fait que chaque sous-unité peut revêtir plusieurs formes. Les sous-unités $\alpha$, spécifiques de chaque protéine $\mathrm{G}$, contiennent le site qui lie le nucléotide et sont dotées d'une activité GTPase. On a identifié au moins neuf gènes différents codant pour une sous-unité $\alpha$. Parmi eux il faut faire une place à part à deux protéines $\mathrm{G}$ qui contrôlent le système de l'adénylate cyclase sensible aux hormones qui catalysent la synthèse de l'AMP cyclique : une Gs qui stimule cette synthèse, et une (ou plusieurs) Gi qui l'inhibe(nt). Or une baisse de la quantité (ou de l'expression) de la sous-unité $\alpha(\mathrm{Gs} \alpha)$ a été observée dans des cellules en culture provenant de sujets atteints d'ostéodystrophie d'Albright. Les principaux symptômes de cette maladie sont la petitesse de la taille, une brachydactylie et des ossifications sous-cutanées. Chez certains malades, de plus, on observe une résistance à l'hormone parathyroïdienne ainsi qu'à d'autres hormones capables de stimuler l'adénylate cyclase. Ce syndrome est appelé pseudohypoparathyroïdisme du fait qu'il existe, non un déficit de l'hormone, mais une résistance à son action. Chez d'autres malades, en revanche, et

même baisse de la Gs $\alpha$ ne s'accompagne pas d'une résistance aux hormones, situation qui a hérité du nom de pseudo-pseudohypoparathyroidisme. Le syndrome d'Albright présentant une transmission du type dominant autosomique, il était naturel d'explorer les familles qui le présentent.

La famille étudiée par Latten et al. [1] (Baltimore, MD et Providence, RI, USA) comporte une fille atteinte du syndrome complet, sa mère pseudo-pseudohypoparathyroïdienne et son frère indemne; les deux malades avaient une réduction d'environ $50 \%$ de l'activité Gs $\alpha$ des membranes des érythrocytes. Elle se traduit par une baisse de la protéine de $45 \mathrm{kDa}$ reconnue sur immunoblot et par l'apparition d'une bande de plus grande taille à $77 \mathrm{kDa}$. Des anticorps spécifiques de la protéine Gs $\alpha$, appliqués à la protéine $77 \mathrm{kDa}$, en reconnaissaient la portion C-terminale mais non la portion $\mathrm{N}$-terminale. Le gène codant pour Gs $\alpha$ est localisé sur le chromosome 20, s'étend sur environ $20 \mathrm{~kb}$ et possède 15 exons. Puisque l'anomalie semblait concerner la partie $\mathrm{N}$-terminale, les auteurs analysèrent le gène dans la partie correspondant aux deux premiers exons. Un fragment portant un site de restriction anormal pour l'enzyme Ncol fut séquencé ; il montra, comme unique anomalie, une transition $\mathrm{A} \rightarrow \mathrm{G}$ en position +1 d'un des deux allèles, transformant le codon initiateur ATG (met) en GTG (val). L'initiation normale ne peut donc avoir lieu, expliquant l'absence de la partie $\mathrm{N}$ terminale dans cette protéine tronquée. Le codon initiateur ATG sui- vant (après 59 acides aminés) est en phase et sert peut-être de point de départ à la protéine mutée, hypothèse qui ne semble pas encore avoir été testée. Reste à comprendre pourquoi cette protéine est, non pas plus petite, mais plus grande que la protéine normale. Une possibilité serait celle de l'agglutination de deux chaînes entre elles que ne parviendraient pas à rompre les conditions dénaturantes de l'électrophorèse.

Cette découverte d'une anomalie provoquée par une mutation du codon d'initiation fait suite à au moins deux autres exemples, d'abord certains types d' $\alpha$-thalassémie et, récemment, la transition ATG $\rightarrow$ ATA dans le gène de l'ornithine $\delta$-transaminase au cours de la forme libanaise de la dégénérescence chorio-rétinienne [4]. L'anomalie responsable, aisément dépistable grâce à l'enzyme Nco1, n'a pas été retrouvée par les auteurs dans d'autres familles atteintes du même syndrome. Par ailleurs, trois mutations distinctes, non décrites en détail, ont été identifiées dans trois familles [2], et, comme dans le cas de Latten et al., seulement sur un des deux allèles.

En dehors des recherches moléculaires encore en suspens, un problème clinique continue à se poser: la même anomalie moléculaire et une même baisse d'activité de $50 \%$ provoquent chez certains malades seulement, une résistance aux hormones. L'explication la plus simple, mais qui n'est nullement prouvée, serait que, comme par exemple dans les porphyries à hérédité dominante, $50 \%$ d'activité pourraient suffire pour certains sujets et non pour d'autres. 
Les progrès que nous venons d'évoquer ne peuvent que stimuler la recherche sur la pathologie des protéines G. Des lignées cellulaires déficientes ou au contraire hyperactives ont été décrites. Le résultat le plus intéressant a été publié l'an dernier par Landis et al. [3] : dans envion la moitié des cas d'acromégalie tumorale, on trouve une mutation somatique sur un des allèles du gène $G s \alpha$, qui diminue l'activité GTPase et provoque ainsi l'activation constitutive de l'adénylcyclase. L'hormone de croissance est rendue indépendante de sa stimuline et fonctionne en permanence. On peut donc espérer découvrir à l'avenir d'autres syndromes résultant de la dysrégulation de Gs $\alpha$ ainsi que d'autres sous-unités des protéines $G$.

\section{J.C.D.}

1. Patten JL, Johns DR, Valle D, et al. Mutation in the gene encoding the stimulatory $G$ protein of adenylate cyclase in Albright's hereditary osteodystrophy. New Engl J Med 1990 ; 322 : $1412-9$

2. Spiegel AM. Albright's hereditary osteodystrophy and defective G proteins. New EnglJ Med 1990 ; 322 : 1461-2

3. Landis CA, Masters SB, Spada A, Pace AM, Bourne HR, Vallar L. GTPase inhibiting mutations activate the alpha chain of Gs and stimulate adenyl-cyclase in human pituitary tumours. Nature 1989: 340 : 692-6.

4. Mitchell GA, Brody LC, Loonet J, et al. An initiator codon mutation in ornithineaminotransferase causing gyrate atrophy of the choroid and retina. $J$ Clin Invest $1988 ; 81$ : 630-3

\section{BRÈVES}

Le récepteur du GM-CSF : premier gène fonctionnel connu de la région pseudo-autosomique des chromosomes $\mathbf{X}$ et $\mathbf{Y}$. Les chromosomes scxuels des mammifères partagent sur leur bras court une petite région terminale de séquences homologues d'ADN, qui s'apparient et se recombinent au cours de la méiose mâle $\left(\mathrm{m} / \mathrm{s} n^{\circ} 6\right.$, vol. 1, p. $331 ; n^{\circ} 3$, vol. 2, p. 157 ; $n^{\circ} 4$, vol. 3, p. 238). Sa longueur est estimée à environ $2500 \mathrm{kpb}$. La transmission des gènes qui la jalonnent est ditc pscudoautosomique. On connaît dans cette zone la localisation de "sondes anonymes" qui permettent de fixer des repères, et, à sa limite, un antigène dc surfacc, MIC2, dont la fonction est inconnue. On ne connaissait, jusqu'à présent, aucun gène fonctionnel chez l'homme, alors quc, chcz la souris, la stéroïde sulfatase y est incluse. C'est dire l'intérêt de la découvcrtc, par une équipe australienne, de la localisation du gène du réccptcur du GM-CSF dans cette région [1]

Le granulocyte-macrophage colony stimulating factor ou GM-CSF agit sur la production des granulocytes et des macrophages par l'intermédiaire d'un récepteur dont l'ADNc a été récemment cloné, répondant à un gène d'environ $50 \mathrm{~kb}$.Gough, et al. [1] en ont recherché la position chromoso- mique par la méthode des hybrides somatiques homme-souris. La conclusion fut que le gène est porté par lc chromosome X. Mais les auteurs ont été frappés par le fait qu'une analyse du gène par polymorphisme de restriction montre souvent deux allèles dans le sexe masculin, suggérant que le chromosome $\mathrm{Y}$ est également porteur du gène. La confirmation fut obtenuc par hybridation in situ, plaçant les gènes du récepteur en Xp21-pter et Ypter-p112. Restait, pour prouver sans équivoque que la transmission est bien pseudoautosomique, à montrer l'existence d'échanges entre $\mathrm{X}$ et $\mathrm{Y}$ à la méiose, par exemple une fille recevant l'allèle de l'Y paterncl, ou un fils héritant de celui de l'X paternel. Sur 14 méioses mâles informatives étudiées dans trois familles, 3 recombinaisons furent observées, une proportion obtenue pour plusieurs sondes anonymes, et beaucoup plus élevée que les 2,5\% de MIC2. Ce dernier locus est donc proche de la limite de la région pseudoautosomique, et le locus du récepteur du GM-CSF lui est distal.

Il s'agit donc bien d'un événement dans l'histoire de la génétique des chromosomes sexuels. Cette découverte pourrait en outre expliquer un phénomène pathologique encore mystérieux. Dans un seul des soustypes (M2) des leucémies aigües myé- loblastiques, on observe une perte d'un $\mathrm{X}$ ou de l'Y dans $25 \%$ des cas. Lc gènc qu'on supposc impliqué dans la genèse de la maladie devrait se situer dans la région pscudoautosomique, puisque le même effet est produit par la perte d'un $\mathrm{X}$ ou $\mathrm{dc}$ l'Y. Les auteurs [1] font donc l'hypothèse que c'est l'inactivation d'un, ou des deux allèles du récepteur du GM-CSF qui scrait en causc. Cette disparition rendrait le clone muté incapable de répondre au GM-CSF. A l'appui de cette thèse, on sait qu'un certain nombre de ces leucémies sont insensibles à l'action du GM-CSF

[Gough NM, et al. Nature 1990 $345:$ : 734-6.]

La spécificité tissulaire de l'aldostérone : l'exemple du tube rénal. L'aldostérone et les glucocorticoïdes ont la même affinité in vitro pour le récepteur minéralocorticoïde ; en revanche, in vivo, la liaison spécifique de l'aldostérone à cc récepteur est bien plus élevée que celle des glucocorticoïdes. Cette spécificité tissulaire est attribuée à l'activité d'une enzyme, la $11 \beta$-hydroxystéroïdc déshydrogénase (11 $\beta \mathrm{OH} \mathrm{SD})$, responsable de la conversion du cortisol (ou de la corticostérone) en cortisone ou autrcs dérivés qui ont unc bien moindre affinité pour le récepteur minéralocorticoïde. Ainsi l'acti- 


\section{BRÈVES 口n}

vité de la $11 \beta \mathrm{OH}$ SD détermine la spécificité tissulaire de l'aldostérone : dans les tissus où l'aldostérone se lie et entraîne des effets biologiques, l'activité $11 \beta \mathrm{OH}$ SD est élevéc (par exemple, rein, parotide et colon), protégcant le récepteur de la fixation des glucocorticoïdes alors qu'elle est basse dans d'autres tissus comme l'hippocampe ou le cœur, où l'aldostérone sc fixe mais n'exerce pas d'effet bien défini [1]. Comme le tubule rénal comporte des segments sensibles (le canal collecteur cortical) et insensibles (le tube proximal) à l'aldostérone, Bonvalet et al. [2] ont cherché à localiser l'activité $11 \beta \mathrm{OH}$ $\mathrm{SD}$ dans des segments tubulaires bien définis de lapin, obtenus par microdissection. Les segments ont été incubés, en présence de ${ }^{3} \mathrm{H}$-corticostéronc $\left({ }^{3} \mathrm{H}-\mathrm{B}\right)$ et les quantités de ${ }^{3} \mathrm{H}-\mathrm{B}$ et de son métabolite ${ }^{3} \mathrm{H}-\mathrm{A}$ ont été déterminées par HPLC. Dans le tube proximal, ${ }^{3} \mathrm{H}$-A représente $19,6 \%$ du total ${ }^{3} \mathrm{H}-\mathrm{A}+{ }^{3} \mathrm{H}-\mathrm{B}$. Dans le canal collecteur cortical, ${ }^{3} \mathrm{H}-\mathrm{A}$ représente $74,1 \%$ du total ; l'activité $11 \beta$ $\mathrm{OH} \mathrm{SD}$ est également élevéc dans d'autres segments tubulaires sensibles à l'aldostérone. L'absence ou l'inhibition de la $11 \beta \mathrm{OH}$ SD peut être observéc chez l'homme et entraîne une hypertension artérielle, une rétention sodée, une hypokaliémie et la suppression du système rénincangiotensine-aldostérone. C'est ce qu'on observe dans le syndrome dit d'excès apparent de minéralocorticoïdes ou au cours de l'administration de quantités importantes de réglisse ou de carbénoxolone, un dérivé de l'acide glycyrrhétinique, principe actif de la réglisse. Dans ces cas, le cortisol présent en concentrations excessives exerce ses effets sur le rein, à la place de l'aldostérone. Dans leur étude, Bonvalet et al. ont utilisé in vitro la carbénoxolone pour inhiber presque complètement la conversion de ${ }^{3} \mathrm{H}-\mathrm{B}$ en ${ }^{3} \mathrm{H}-\mathrm{A}$. Ainsi, l'activité $11 \beta \mathrm{OH}$ SD est élevéc dans les segments du tube rénal sensibles à l'aldostérone et basse dans les segments insensibles. Comme dans d'autres tissus, cette activité enzyma- tique joue un rôle important pour déterminer la spécificité du site d'action de l'aldostérone le long du néphron chez le lapin.

[1. Funder JW, et al. Science 1988 ; 242: 582-5.]

[2. Bonvalet JP, et al. J Clin Invest 1990. Sous presse.]

\section{L'extraordinaire diversité de} la vie bactérienne. La méthode classique permettant de caractériser les différentes espèces de microorganismes consiste à les cultiver... cc qui revient à méconnaître celles qui sont récalcitrantes à se multiplier dans les milieux synthétiques que leur offrent les expérimentateurs. Plusicurs équipes - dont une du Montana et l'autre de l'Oregon, aux États-Unis, viennent de publier leurs travaux [1, 2] - ont donc cherché à approcher la diversité des microorganismes sans recourir aux procédés de culture. Elles ont ainsi isolé l'ARN total de " niches écologiques" riches en micro-organismes, l'ont recopié en $\mathrm{ADNc}$ qui a ensuitc été cloné. Les amorces utilisées pour la synthèse du premier brin d'ADNc étaient spécifiques d'une région universellement conservéc des ARN ribosomiques $16 \mathrm{~S}$. Différents clones d'ADNc de ces $A R N$ ribosomiques ont alors été séquencés au hasard. Sur 14 séquences issues de cyanobactérics vivant à $55^{\circ} \mathrm{C}$ dans le parc de Yellowstone, 8 correspondaient nettement à des espèces différentes... dont aucune n'appartenait aux espèces déjà identifiées après culture [1]. Des résultats du même type ont été rapportés avec du bactérioplancton de la mer des Sargasses [2]. La diversité des micro-organismes vivants est donc encore bien plus grande que cela n'était soupçonné jusqu'à présent.

[1. Ward DM, et al. Nature 1990 ; $345: 63-5$.

[2. Giovannoni SJ, et al. Nature 1990 ; 345 : 60-3.]

Hémophilie de type $B$ et mutations du promoteur du facteur
IX. L'origine moléculaire de la déficience en facteur IX dans certains cas d'hémopholie de type B (Leyden) vient d'être rapportéc [1]. Chez ces malades, plusieurs mutations ponctuclles sont retrouvées dans la séquence du promoteur du gène de ce facteur de coagulation. Une de ces mutations a été localisée dans la séquence cible du facteur transcriptionncl $\mathrm{C} / \mathrm{EBP}$ qui joue un rôle important dans l'expression de nombreux gènes hépatiques $\left(m / s, n^{\circ} 5\right.$, vol. 6, p. 486).

Ce facteur ne peut pas se lier à la séquence mutée et n'active plus la transcription du gène du facteur IX. Ceci pourrait expliquer la diminution de plus de $90 \%$ de la concentration du facteur IX dans le sérum. I a réapparition de ce facteur dans le sérum à la puberté pourrait être expliquéc par une augmentation de l'expression de son gène probablement due aux modifications hormonales. Ces résultats illustrent l'importance de la protéine C/EBP dans l'expression du facteur IX ct constituent un nouvel exemple de maladies héréditaires dues à une mutation dans le promotcur d'un gène [2]. [1. Crossley M, et al. Nature 1990 ; 345 : 444-6.]

12. Martin DIK, et al. Nature 1989 ; 338 : 435-8.]

Le gène $d u$ syndrome d'Alport est codé par la chaîne $\alpha 5$ (IV) du collagène. Le syndrome d'Alport est une affection liée au sexe qui se caractérise par une glomérulonéphrite progressive associée à une surdité. Cette maladie est fréquente (environ 1 garçon atteint sur 5000 naissances) et de gravité variable selon les familles. Des études immunologiques ont démontré qu'une chaîne de type $\alpha$ du collagène de type IV, spécifique des membranes basales, était anormale chez les malades. Des efforts considérables ont alors été consentis pour rechercher une chaîne de collagène $\alpha(\mathrm{IV})$ codée par un gène situé sur le chromosome $\mathrm{X}\left(\mathrm{m} / \mathrm{s}, n^{\circ} 6\right.$, vol. 6, p. 601). Le gène 


\section{DRÈVES DI}

COL4A5 a ainsi été identifié ; localisé en Xq22, il code pour une nouvelle espèce de collagène, la chaîne $\alpha 5$ (IV). Des chercheurs associés de Oulu en Finlande et de Salt Lake City (UT) et Rochester (NY) aux USA viennent maintenant de détecter trois types de mutation de ce gène COL4A5 dans des familles de malades atteints du syndrome d'Alport [1]. Ce gène est donc bien celui qui est impliqué dans une partie... ou la totalité des observations de cette maladie.

[1. Barker DF, et al. Science 1990 ; 248: 1224-7.]

LE récepteur T (TcR) de la sclérose en plaque? Des clones de cellules $\mathrm{T}$ autoréactives contre un peptide immunodominant de la protéine basique de la myéline ont été isolés chez des malades atteints de sclérose en plaque. Alors que la fréquence de l'utilisation de la région variable $\mathrm{V} \beta 17$ de la chaîne $\beta$ du $\mathrm{TcR}$ est de $9 \%$ dans des clones variés de lymphocytes $\mathrm{T}$, elle est de $56 \%$ dans les clones réagissant avec un peptide allant de la position 84 à la position 102 de la protéine basique de la myéline [1]. Une autre étude, portant sur les chaînes $\alpha$ des lymphocytes $\mathrm{T}$ infiltrant le cerveau des malades, va également dans le sens d'une utilisation d'un petit nombre seulement de segments variables $\mathrm{V} \alpha$ [2]. Si la sclérose en plaque se révèle bien être une maladie autoimmune due à l'intervention de clones de cellules $\mathrm{T}$ autoréactives possédant un petit nombre de types de récepteur, une immunomodulation spécifique peut s'envisager, soit par immunodestruction spécifique des lymphocytes autoréactifs, soit par utilisation de "leurres" antigéniques bloquant la reconnaissance par ces cellules cytotoxiques de leur cible.

[1. Wucherpfennig KW, et al. Science 1990 ; 248 : 1016-9.]

[2. Oksenberg JR, et al. Nature $1990 ; 345: 344-6$.]

$\mathrm{m} / \mathrm{s} n^{\circ} 7$ vol. 6 , septembre 90
FLASH

\section{G COMME CANCER}

Les lecteurs de $\mathrm{m} / \mathrm{s}$ ont pu trouver, au fil des numéros, des informations concernant la protéine Ras et le cancer, les mutations de Gprotéines dans des cancers endocriniens, le rôle possible de GDP-kinase dans le pouvoir métastatique des cellules. Ils ont aussi appris qu'un homologue de ras, rap-1/Krev-1, était un potentiel anti-oncogène. Ils apprendront maintenant avec surprise que le gène de susceptibilité à la neurofibromatose de type 1, dont Jean-Claude Dreyfus racontera la découverte dans le prochain numéro de $\mathrm{m} / \mathrm{s}$, code pour une protéine présentant de nettes analogies de séquence et de structure globale avec la protéine GAP (GTPase activating protein), stimulant I'hydrolyse du GTP fixé sur la protéine Ras, et par conséquent l'inactivant. Les Gprotéines sont des intermédiaires tellement ubiquitaires des processus de signalisation qu'il n'est probablement pas stupéfiant, en fait, de les trouver impliquées à différents niveaux du contrôle de la prolifération cellulaire. Dans le cas du gène NF1, on peut imaginer que l'absence d'une protéine de type GAP, incapable d'inactiver une Gprotéine de type Ras, conduise à une croissance incontrôlée.

Source : Cell. 1990 ; 62 : 599-608.

A. $K$.

\section{Erratum :}

Les onze premières références bibliographiques de l'article de Claude Hamelin, paru dans médecine/sciences $n^{\circ} 6$, vol. 6, de juin-juillet 1990, pp. 544-51, ont malencontreusement été remplacées par d'autres références n'appartenant pas à cet article. Les références correctes étaient :

1. Apperley JF, Goldman JM. Cytomegalovirus: biology, clinical features and methods for diagnostic. Bone Marrow Transpl $1988 ; 3: 253-64$.

2. Forbes BA. Acquisition of cytomegalovirus infection : an update. Clin Microbiol Rev 1989 ; 2 : 204-16.

3. Meyers JD. Management of cytomegalovirus infection. $\mathrm{Am} J \mathrm{Med} 1988 ; 85$ : 102-6.

4. Yow MD. Congenital cytomegalovirus disease : a NOW problem. I Infect Dis $1989 ; 159: 163-7$

5. Landini M-P, Michelson S. Human cytomegalovirus proteins. Prog Med Virol $1988 ; 35: 152-85$.

6. Scott AA, Walker KA, Hennigar LM, et al. Detection of cytomegalovirus in shell vial cultures by using a DNA probe and early nuclear antigen monoclonal antibody. J Clin Microbiol $1988 ; 26: 1895-7$.

7. Sorbello, AF, Elmendorf SL, McSharry, et al. Rapid detection of cytomegalovirus by fluorescent monoclonal antibody staining and in situ DNA hybridization in a dram vial cell culture system. J Clin Microbiol $1988 ; 26: 111-4$.

8. Bale JF Jr, O'Neil BE, Hart MN, et al. Human cytomegalovirus nucleic acids in tissues from congenitally infected infants. Pediat Neurol 1989; 5 : 216-20.

9. Jiwa NM, Raap AK, Van de Rijke, et al. Detection of cytomegalovirus antigens and DNA in tissues fixed in formaldehyde. $J$ Clin Pathol 1989; 42 : 749-54.

10. Hsia K, Spector DH, Lawrie J, et al. Enzymatic amplification of human cytomegalovirus sequences by polymerase chain reaction. J Clin Microbiol 1989 ; 27 : 1802-9.

11. Jiwa NM, Van Gemert GW, Raap $\mathrm{AK}$, et al. Rapid detection of human cytomegalovirus DNA in peripheral blood leukocytes of viremic transplant recipients by the polymerase cahin reaction. Transplantation $1989 ; 48: 72-6$.

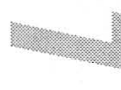

\section{3}

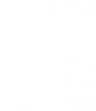
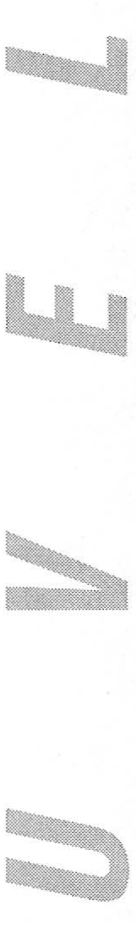